\title{
Risk stratification and mortality prediction in Falciparum Malaria
}

\author{
Khadanga $\mathbf{S}^{1}$ \\ ${ }^{1}$ Dr. Sagar Khadanga. Assistant Professor, Department of General Medicine, AIIMS, Bhopal, Madhya Pradesh, India.
}

Address for correspondence: Dr. Sagar Khadanga, E mail- sagarkhadanga@yahoo.com

\begin{abstract}
Falciparum malaria has remained an important public health problem in India over the years. WHO enumerates a list of complications to diagnose severe falciparum malaria which was initially provided in 1990 and then revised in 2000. But the importance and prognosis of each complication has not been assigned. Various strategies have been employed for risk stratification in falciparum malaria by various authors in different parts of world. Here we would like to discuss the available literatures from India and some accomplished western literatures to prognosticate severe falciparum malaria patients.
\end{abstract}

Keywords: Falciparum malaria, Cerebral malaria, Glasgow coma scale, Jaundice, Renal failure.

\section{Introduction}

Malaria is an important cause of morbidity and mortality in both children and adults all over the world. More than two third of total malaria cases are reported from Africa and South East Asian Region. Of the 34 million reported cases of malaria in South East Asian Region, India reports approximately two-third of all confirmed cases. In India 5 states account for more than $60 \%$ of these cases: Odisha, Chhattisgarh, Madhya Pradesh, Jharkhand and West Bengal [1]. Around 50\% of the total malaria cases reported in India are due to Plasmodium falciparum [2]. There was a need to identify the severe cases in falciparum malaria where the mortality is high. WHO in 1990 published the criteria to identify the severe cases [3].

But the importance of each of the complication was not addressed and the clinicians faced great difficulties in day today practice.

So the clinicians all over the world tried to formulate something which can address this problem better. In 2000 WHO again published the guidelines to identify severe falciparum malaria with some modification but the problem remained as it was [4].

There are lots of researches regarding the prognostication of severe falciparum malaria. Some has been too simple based on clinical parameters only and some are more really complex requiring sophisticated laboratory.

Some of them have been validated by other authors in different patient profile and many of them are not validated by others.

We will discuss here some of the easy to use literatures from India and abroad which can influence our clinical practice.
Malaria Severity Assessment Score (MSA) for adults by Mishra et al [5]

The score is based on four parameters namely severe anemia, acute renal failure, respiratory distress and cerebral malaria. Points are given to each of the parameter and the final score is calculated adding all the four points. Thus the Malaria Score for Adults $($ MSA) $=1$ (severe anaemia) +2 (acute renal failure) +3 (Respiratory distress) +4 (cerebral malaria). The MSA ranges from 0 to 10 . The mortality was $2 \%$ for MSA $0-2 ; 10 \%$ for MSA 3-4, 40\% for MSA 5-6 and 90\% for MSA 7 or more. The sensitivity is claimed to be $89.9 \%$, specificity $70.6 \%$ and positive predictive value $94.1 \%$ when 5 is taken as the cut off value by the original authors. This appears to be one of the simplest method of risk stratification.

\section{APACHE II by Wilairatana et al [6]}

APACHE-II (Acute Physiology and Chronic Health Evaluation) score is more popularly applied in patients of acute critical illness. APACHE II score is the sum of the acute physiology score (vital signs, oxygenation, laboratory values), Glasgow coma score, age and chronic health points. Wilairatana et al in Bangkok, Thailand applied this score to stratify the prognosis in patients of cerebral malaria. With the cutoff point at a score of 24 , the APACHE-II stratified the patient's mortality outcome with $95.8 \%$ accuracy. This appears to be most sensitive but with low specificity.

\section{Clinical Scoring Index by Teano $R$ et al [7]}

Teano $\mathrm{R}$ et al. proposed a Clinical Scoring Index for predicting outcome in cerebral malaria with a possible score of 0-14. Level of consciousness, multiple convulsion, labored respiration, circulatory collapse and 
Editorial

abnormal bleeding were the parameters taken for calculating the score. With an optimum score of 7 , it could predict the mortality with a sensitivity of $92 \%$ and specificity of $95 \%$.

\section{Malaria Severity Score by Mohapatra MK et al [8]}

The authors devised Malaria Severity Score for severity assessment and risk prediction of hospital mortality for falciparum malaria in adults. There is a score for each organ dysfunction according to its severity level and for each score there is a probability of mortality (table-2,3). This appears to be a very good risk stratification scoring system but it can have observer bias and a bit cumbersome to remember for day to day practice.

\section{GCBRS score by Mohapatro BN et al [9]}

There are five parameters and the initial alphabet of each parameter are given the pneumonic of GCRBS. Glasgow coma score $(\mathrm{GCS}<11)$, Creatinine $(>3 \mathrm{mg} / \mathrm{dl})$, Respiratory rate $(>24$ per minute), Bilurubin $(>10 \mathrm{mg} / \mathrm{dl})$ and Systolic BP $(<90 \mathrm{mmHg})$ are taken into account. Out of these parameters, three can be identified at bedside and rest two requires simple biochemical analysis. The GCRBS score has a possible score of $0-10$. Higher the score poorer is the outcome. At a cutoff score of 5 the prediction of mortality has a sensitivity of $85.3 \%$ and specificity of $95.6 \%$. The greatest advantage of this scoring system is that all the parameters are objective and has very minimal to none observer bias (Table-4).

\section{Conclusion}

Risk stratification and mortality prediction in severe falciparum malaria is the need of the hour. Of the above mentioned parameters any one of the scoring system can be applied and none has a clear cut advantage over the other. The GCRBS score seems to a very good working tool as it is very easy to calculate, easy to remember and has been developed in India. But it has to stand tall over the period of time and has to be validated in different groups of patients.

Gohiya $\mathrm{P}[10]$ et al in this issue assessed prognostic indicators in falciparum malaria. Biochemical parameters are also very useful in addition to clinical symptoms \& signs.

\section{References}

1.World malaria Report 2010: www. Who.int/malaria/ world_malaria_report_2010/en/index.html

2. Dhingra N, Jha P, Sharma VP, Cohen AA, Jotkar RM, Rodriguez PS, Bassani DG, Suraweera W, Laxminarayan R, Peto R, for the Million Death Study Collaborators: Adult and child malaria mortality in India: a nationally representative mortality survey: Lancet 2010;376: 1768-74.

3. WHO: Severe and complicated malaria. Trans R Soc Trop Med Hyg 1990, 84(suppl 2):1-65. [PubMed]

4. WHO: Severe Falciparum Malaria ; Trans R Soc. Tropical Med Hyg 2000;94:S1-S90. [PubMed]

5. Mishra SK, Panigrahi $\mathrm{P}$, Mishra R, Mohanty S. Prediction of outcome in adults with severe falciparum malaria: a new scoring system. Malaria Journal 2007, 6:24. [PubMed]

6. Knauss WA, Drapper EA, Wagner DP, Zimmerman JE: APACHE II : a severity of disease classification system. Crit Care Med 1985, 13:818-29. [PubMed]

7. Teano R, Robles AM and Dimaano E. A clinical scoring index for predicting outcome in cerebral malaria. Phil Journal Microbiol Infect Dis 2003;32: 43-44.

8. Mohapatra MK, Das SP. The Malaria Severity Score: A Method for Severity Assessment and Risk Prediction of Hospital Mortality for Falciparum Malaria in Adults. JAPI 2009;57:119-126. [PubMed]

9. Mohapatra BN, Jangid SK, Mohanty R. GCRBS score: A New Scoring System for Predicting Outcome in Severe Falciparum Malaria. JAPI 20014; 62(1):14-17. [PubMed]

10. Gohiya P, Goel M, Dwevedi R, Renwal S. Clinical profile and prognostic indicators of Plasmodium Falciparum Malaria in children. Int J Med Res Rev 2014;2(3):215-220. [PubMed]

\section{How to cite this article?}

Khadanga S. Risk stratification and mortality prediction in Falciparum Malaria. Int J Med Res Rev 2014;2(3): 176-177.doi:10.17511/ijmrr.2014.103.01 